\title{
Investigating Electromagnetic Material Properties of Collagen at THz for Health Monitoring Applications
}

\author{
Nishtha Chopra \\ School of Electronics Engineering \\ And Computer Science \\ Queen Mary University of London \\ Mile End Road, E1 4NS \\ n.chopra@qmul.ac.uk
}

\author{
Akram Alomainy \\ School of Electronics Engineering \\ And Computer Science \\ Queen Mary University of London \\ Mile End Road, E1 4NS \\ a.alomainy@qmul.ac.uk
}

\author{
Mike Philpot \\ Blizard Institute \\ Barts and The London School of \\ Medicine and Dentistry \\ 4 Newark Street, London, E1 2AT \\ m.p.philpott@qmul.ac.uk
}

\begin{abstract}
The elegant concept of Nanocommunication at terahertz (THz) frequency is proposed with its main focus on investigating electromagnetic material properties of synthesized Dermis layer of the skin via THz-Time Domain Spectroscopy (TDS). The paper highlights the study of human skin and cell culture. Methods are applied to synthesize collagen and measurements are carried out in the $\mathrm{THz}$ band ranging from $0.1-3 \mathrm{THz}$. The results are further proposed for Nanonetwork channel propagation models and transmission schemes based on $\mathrm{THz}$ electromagnetic communication.
\end{abstract}

\section{Keywords}

Terahertz Time domain Spectroscopy, refractive index, collagen, electromagnetic communication, nano-networks, health monitoring

\section{INTRODUCTION}

$\mathrm{THz}$ spectroscopy has found its niche in medical diagnosis [1,2], imaging [3,4] and in depth study of biomolecules [5] owing to its unique spectral features explaining the orientation, rotational \& translational motion and structural assembly of the biomolecules. These non-ionizing radiations [6,7] with a penetration depth of ranging from hundreds of $\mu \mathrm{m}$ to few tens of $\mathrm{mm}$ are an emerging concept for new generation healthcare devices. The proposed electromagnetic (EM) based communication in the terahertz band is considered a viable technique for supporting data exchange in the Internet of Nano-Things (IoNT) . Thus, the study of wireless transmission models at the nano-scale should be carefully addressed to sustain the progress of this technology [8]. Thorough studies have been carried out from modeling such communication channels in the atmospheric medium [9-12], however there is a huge gap of information in regard to electrometric behavior in the biological medium. Many papers have claimed the results for THz-TDS of various excised tissues [13-15] in the THz band but lack consistency. In the previous paper [8], path loss and molecular absorption noise temperature have been obtained from optical parameters of human skin tissues, verified through extensive experimental tests.
Results of this investigation clearly show that, human tissues strongly hinder EM communications in the terahertz band and novel communication schema are strongly required to enable intra-body nanocommunication based systems. Hence to establish the new and improved healthcare diagnostic system based on the trails of Internet of Nano-Things, a meticulous study of electromagnetic material properties of tissues is proposed in this paper.

\section{THZ TIME DOMAIN SPECTROSCOPY - OPERATION \& EXPERIMENTAL USABILITY}

The extraction of complex dielectric properties of biological material became evident with the handiness of $\mathrm{THz}$ Time Domain Spectroscopy (THz-TDS). It is a scheme of coherent generation and detection of ultrafast laser pulses focused via set of off-axis parabolic mirrors on the desired sample. Depending on the size of the sample, it is either collimated or focused by these mirrors. The titanium-Sapphire laser system produces femtosecond pulses, which are then divaricated with the aid of a beam splitter into two beams namely: pump and probe [FIG. 1]. These two beams together but operating in separate directions form the basis of detection and generation of $\mathrm{THz}$ radiation. Generation is made possible with the usability of photoconductive antenna illuminated by the pump beam resulting in a transient photocurrent. The pump beam travels through a time-delay stage resulting in a delay of $\Delta \mathrm{t}$ relative to probe beam [16]. Thus THz-TDS spectroscopy provides direct estimation of both phase and magnitude of the sample unlike its contemporary schemes like FTIR or Raman Spectroscopy [17].

On the same principle of operation THz-TDS installed at Queen Mary University of London, was utilized for experimental verification of the dielectric properties of biological samples. The system offers a sophisticated range of $0.1-4 \mathrm{THz}$ thus providing an opportunity of broader spectral analysis. The pulsed laser -Ti: Sapphire has an adjustable wavelength range of 750-850 nm; repetition rate of $80 \mathrm{MHz}$ and peak power of about $1 \mathrm{~W}$. The mechanical delay stage which is responsible for providing the time-delay between the two beams, has maximum travel distance of $15 \mathrm{~cm}$. THz emitter is LT-GaAs photoconductive antenna with a biased voltage of $200 \mathrm{~V}$ and a gap size of approximately $0.5 \mathrm{~mm}$ which makes the laser beam positioning easier. A ZnTe crystal is employed with thickness of $2 \mathrm{~mm}$, which allows enough interaction length of probe beam and $\mathrm{THz}$ wave in the crystal. 


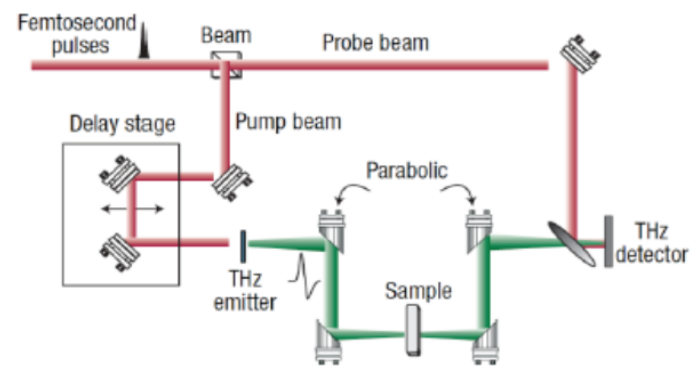

Fig. 1 Schematic Of THz system [15]

\section{SAMPLE PREPARATION}

Analogous to the human skin tissue, artificial synthesis of collagen was done in a controlled environment at Subcutaneous Group Blizard Institute, Queen Mary University of London. Collagen forms the second layer of human skin which the dermis and is thickest most layer of the skin. It provides scaffolding for essential cells and structures including blood vessels, lymph vessels, hair follicles and sweat glands. The thickness of this layer varies from $1.5 \mathrm{~mm}$ to $4 \mathrm{~mm}$ [18]. Collagen type 1 is extracted from the rat-tail is a heterotrimer consisting of two (alpha-1) chains and one (alpha-2) chains. [19]. Cross-linking of molecules are critical for mechanical integrity of collagen, this could account for the weaker mechanical properties of tissue at the larger length.
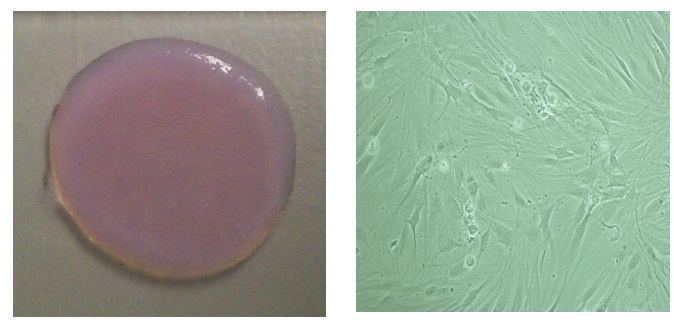

Fig.2 (left) Artificially synthesized collagen layer at the Blizard Institute, QMUL \& (right) Fibroblast cells assisting the growth of collagen layer.

Collagens can be readily isolated from animal tissues, stored in solution or as a solid, and used as biocompatible coatings or softtissue substitutes that can protect and support damaged tissues [19]. The synthesis of collagen [FIG. 2] was done with a help of standard recipe with total stock solution of $3.35 \mathrm{mg} / \mathrm{ml}$ out of which $80 \%$ is collagen. The solution was thoroughly mixed and separated into sections of 6 -well plate. The gels are incubated $(5 \%$ $\mathrm{CO}_{2}$ ) at $37^{\circ} \mathrm{C}$ to support the growth of the cells. The samples were wedged between first a TPX window with a spacer of $0.2 \mathrm{~mm}$, thus keeping the thickness of the sample constant.

\section{DATA ANALYSIS}

The complex dielectric constant of a sample can then be determined by deconvoluting the reference from the sample measurement [23]. A detailed temporal scan of a $\mathrm{THz}$ waveform is achieved as a result of discrete translational stage controlling the optical delay line. This waveform, or a time- resolved $\mathrm{THz}$ pulse, is then readily convertible to its corresponding $\mathrm{THz}$ wave spectrum via a Fourier transform [20]. A broadband $\mathrm{THz}$ signal transmitted through the sample is influenced by its absorption and dispersion. This results in change of transmitted signal thus giving a way to extract intrinsic properties of the sample at certain discrete frequencies. This response can be described in terms of a complex refractive index [22]. However, other factors also contribute to the modification of the amplitude and phase of the signal - such as the shape and surface morphology of a sample under measurement, surrounding atmosphere and the $\mathrm{THz}$ propagation path. Thus, in order to attain a complex refractive index from the measurement, it requires the geometrical analysis of wave propagation along with a reference measurement [21].

\section{RESULTS \& DISCUSSION}

Understanding the propagation of transmitted wave is done by TDS data processing. It is the summation of waves propagating through the air - sample (or) in some cases air-sample holdersample. The sample spectrum is measured at a normal incidence and can be expressed as:

$E_{\text {samp }}(\omega)=\eta \frac{4 \hat{n}_{S} n_{\circ}}{\left[\hat{n}_{S}+n_{\circ}\right]^{2}} \exp \left\{-j \hat{n}_{s} \frac{\omega l}{c}\right\} E_{\circ}(\omega)$

where $\eta$ is the transmission factor for free space surrounding the sample; $\hat{n}_{s}=n_{\mathrm{s}}+j k_{\mathrm{s}}$ is the complex refractive index of the sample; $n 0$ is the refractive index of air; and $l$ is the sample thickness for a normal angle of incidence [20]. Similary, the reference spectrum can be given as:

$E_{\text {ref }}(\omega)=\eta \exp \left\{-j n_{\circ} \frac{\omega l}{c}\right\} E_{\circ}(\omega)$

The value phase of sample spectrum can be subtracted from that of reference to provide the refractive index. Reference spectrum is the propagation of $\mathrm{THz}$ wave through the empty sample holder i.e. without any sample. The material parameter extraction process requires these two spectra, $E_{\text {samp }}(\omega)$ and $E_{\text {ref }}(\omega)$, which are Fourier transformed from time-domain measurements.

Normalizing the sample spectrum by the reference, or $E_{\text {samp }}(\omega) /$ $E_{\text {ref }}(\omega)$ yields the complex transfer function of the sample in the frequency domain [21]:

$$
H_{\circ}(w)=\frac{4 \hat{n}_{s} n_{\circ}}{\left[\hat{n}_{s}+n_{\circ}\right]^{2}} \exp \left\{-j \hat{n}_{s} \frac{\omega l}{c}\right\} \exp \left\{-j\left[-n_{\circ}\right] \frac{\omega l}{c}\right\}
$$

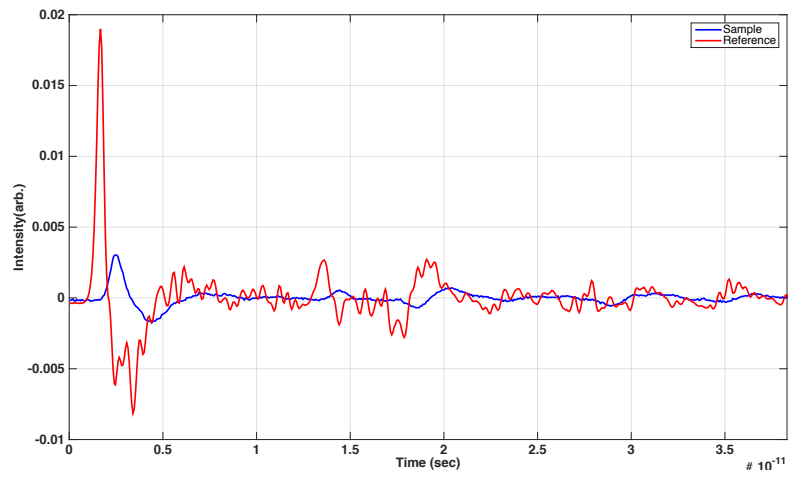

Fig. 3 THz-TDS spectra of sample and reference, as illustrated the blue colored plot corresponding to collagen has significantly large attenuation in comparison to the reference plot. However, a defined shift can be observed owing to sample's response to the incoming $\mathrm{THz}$ radiation. 


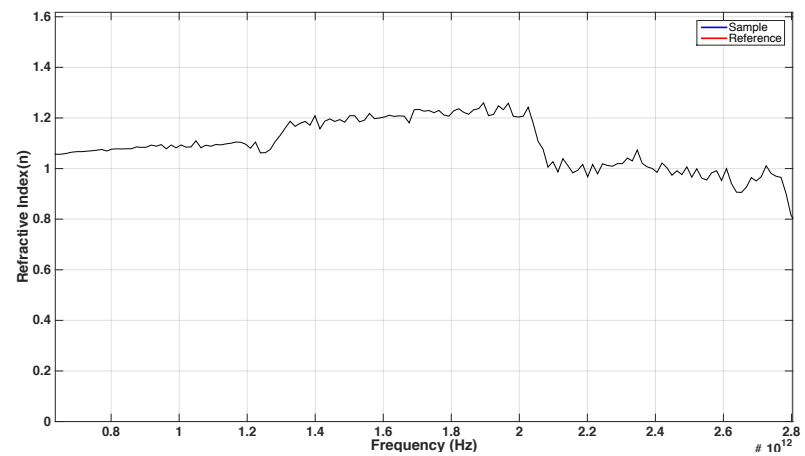

Fig.4 Refractive index of the sample - collagen of $0.2 \mathrm{~mm}$ thickness is estimated to be 1.1 , which is in sync with previously published work [13].

\section{CONCLUSION}

In this paper, we investigated the electromagnetic material properties of collagen, which is artificially synthesized. Minor differences in the results when compared to already published data is due to the varied sample preparation techniques and data processing. Future work holds for more consistent data and inclusion of uncertainties in data processing. The present data and method is a stepping-stone towards establishing new generation nanocommunication based health diagnostic devices. Further, the refractive index value will be utilized to develop a dielectric model [22] for biological materials. This dielectric model will be then utilized for channel propagation models and transmission schemes.

\section{ACKNOWLEDGMENTS}

Institute of Bioengineering PhD Scholarship, QMUL and Parts of this publication, specifically Section 2, were made possible by NPRP grant \# NPRP 7-125-2-061 from the Qatar National Research Fund (a member of Qatar Foundation).

\section{REFERENCES}

[1] Gian Piero Gallerano, " THz radiation in biological reseach, investigation on diagnostic and study on potential genotoxic effects - THz bridge report", May 2004

[2] V P Wallace, B C Cole, R M Woodward, R J Pyel, and D A Amone. Biomedical applications of terahertz technology. IEEE, pages 308-309, 2002.

[3] E. Berrya and et al., "Optical properties of tissue measured using terahertz pulsed imaging," in Proceedings of SPIE: Physics of Medical Imaging, 2003.

[4] W L Chan, J Deibel, and D M Mittleman. Imaging with terahertz radiation. Rep.Prog. Phys., 70:1325-1379, 2007.

[5] Applied Research and Photonics. Terahertz spectroscopy brings new dawn of biological research. available from http://arphotonics.net, 2008.

[6] Gian Piero Gallerano, “ THz radiation in biological research, investigation on diagnostic and study on potential genotoxic effects - THz bridge report”, May 2004
[7] James C. Lin, "Electromagnetic Fields in Biological Systems Book", Taylor \& Francis Inc.

[8] Piro, G.; Ke Yang; Boggia, G.; Chopra, N.; Grieco, L.A.; Alomainy, A., "Terahertz Communications in Human Tissues at the Nanoscale for Healthcare Applications," Nanotechnology, IEEE Transactions on , vol.14, no.3, pp.404,406, May 2015

[9] I. F. Akyildiz, F. Burnetti and C. Blazquez, "Nanonetworks: a new communication paradigm," Computer Networks, vol. 52, pp. 2260-2279, 2008.Ian, guiseppe, bush papers

[10] I. F. Akyildiz and J. M. Jornet, "Electromagnetic Wirelss Nanosensor Networks," Nano Communication Networks, vol. 1, pp. 3-19, 2010.

[11] J.M. Jornet and I.F. Akyildiz, "Channel Modeling and Capacity Analysis for Electromagnetic Wireless Nanonetworks in the Terahertz Band," Wireless Communications, IEEE Trans. on, vol. 10, pp. 3211-3221, 2011.

[12] S.F. Bush, Nanoscale Communication Networks: Artech House, 2010.

[13] Peter M. Corridon ; Ricardo Ascázubi ; Courtney Krest ;Ingrid Wilke; Time-domain terahertz spectroscopy of artificial skin. Proc. SPIE 6080, Advanced Biomedical and Clinical Diagnostic Systems IV, 608007 (February 25, 2006)

[14] A. J. Fitzgerald and et al., "Catalogue of Human Tissue Optical Properties at Terahertz Frequencies," Journal of Biological Physics, vol. 9, pp. 123-128, 2003.

[15] Oleksandr Sushko, , "Terahertz dielectric study of biomolecules using time-domain spectrometry and molecular dynamics simulations", Thesis 2014 Queen Mary University of London

[16] Y-S Lee. Principles of terahertz, science and technology. Springer, 2009.

[17] J L Koenig. Application of fourier transform infrared spectroscopy to chemical systems. Appl. Spectrosc., 29:293-308, 1975.

[18] National Cancer Institute - www.cancer.gov

[19] Chang SW1, Shefelbine SJ, Buehler MJ. Structural and mechanical differences between collagen homo- and heterotrimers: relevance for the molecular origin of brittle bone disease, Biophys J. 2012 Feb 8; 102(3):6408.

[20] T. Dorney, R. Baraniuk, and D. Mittleman, "Material parameter estimation with terahertz time-domain spectroscopy," J. Opt. Soc. Am. A 18, 1562-1571 (2001).

[21] Tych, K.M.; Wood, C.D.; Tych, W., "A Simple TransferFunction-Based Approach for Estimating Material Parameters From Terahertz Time-Domain Data," Photonics Journal, IEEE, vol.6, no.1, pp.1,11, Feb. 2014

[22] Caroline B. Reid, George Reese, Adam P. Gibson and Vincent P. Wallace, "Terahertz time domain spectroscopy of human blood," IEEE JOURNAL OFBIOMEDICAL AND HEALTH INFORMATICS, 
